\title{
Development and Validation of Nomograms to Predict Prognosis of Oral and Oropharyngeal Mucoepidermoid Carcinoma: A SEER-Based Study
}

\section{muyuan liu}

Department of Head and Neck, Cancer Hospital of Shantou University Medical College, Shantou

\section{Litian Tong}

Department of Anesthesiology, Cancer Hospital of Shantou University Medical College, Shantou

\section{Manbin Xu}

Department of Head and Neck, Cancer Hospital of Shantou University Medical College, Shantou

\section{Xiang Xu}

Department of Anesthesiology, Cancer Hospital of Shantou University Medical College, Shantou

\section{Bin Liang}

Department of Cell Biology and Genetics, Key Laboratory of Molecular Biology in High Cancer Incidence Coastal Chaoshan Area of Guangdong Higher Education Institutes, Shantou University Medical College

\section{Xuhong Song}

Department of Cell Biology and Genetics, Key Laboratory of Molecular Biology in High Cancer Incidence Coastal Chaoshan Area of Guangdong Higher Education Institutes, Shantou University Medical College

\section{Lingzhu Xie}

Department of Cell Biology and Genetics, Key Laboratory of Molecular Biology in High Cancer Incidence Coastal Chaoshan Area of Guangdong Higher Education Institutes, Shantou University Medical College

\section{Ting Su}

Department of Cell Biology and Genetics, Key Laboratory of Molecular Biology in High Cancer Incidence Coastal Chaoshan Area of Guangdong Higher Education Institutes, Shantou University Medical College

\section{Hanwei Peng}

Department of Head and Neck, Cancer Hospital of Shantou University Medical College, Shantou

\section{Dongyang Huang ( $\sim$ huangdy@stu.edu.cn )}

Department of Cell Biology and Genetics, Key Laboratory of Molecular Biology in High Cancer Incidence Coastal Chaoshan Area of Guangdong Higher Education Institutes, Shantou University Medical College

\section{Research Article}

Keywords: Oral cavity, Oropharynx, Mucoepidermoid carcinoma, Prognosis, Overall survival, Diseasespecific survival, Nomogram, SEER 
Posted Date: December 15th, 2020

DOI: https://doi.org/10.21203/rs.3.rs-125136/v1

License: (c) (i) This work is licensed under a Creative Commons Attribution 4.0 International License. Read Full License 


\section{Development and Validation of Nomograms to Predict Prognosis of Oral and Oropharyngeal Mucoepidermoid Carcinoma: a SEER-Based Study}

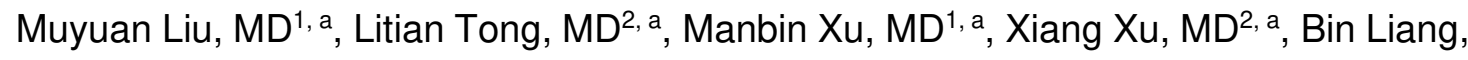
$\mathrm{MD}^{3}$, Xuhong Song, $\mathrm{MD}^{3}$, Lingzhu Xie, $\mathrm{MD}^{3}$, Ting Su, $\mathrm{MD}^{3}$, Hanwei Peng, $\mathrm{MD}^{1,{ }^{*} \text {, }}$ Dongyang Huang, $\mathrm{MD}^{3, *}$

${ }^{1}$ Department of Head and Neck, Cancer Hospital of Shantou University Medical College, Shantou, China

${ }^{2}$ Department of Anesthesiology, Cancer Hospital of Shantou University Medical College, Shantou, China

${ }^{3}$ Department of Cell Biology and Genetics, Key Laboratory of Molecular Biology in High Cancer Incidence Coastal Chaoshan Area of Guangdong Higher Education Institutes, Shantou University Medical College, Shantou, China

${ }^{*}$ Corresponding authors:

Dongyang Huang, Department of Cell Biology and Genetics, Key Laboratory of Molecular Biology in High Cancer Incidence Coastal Chaoshan Area of Guangdong Higher Education Institutes, Shantou University Medical College, Shantou, China. E-mail address: huangdy@stu.edu.cn

Hanwei Peng, Department of Head and Neck, Cancer Hospital of Shantou University Medical College, Shantou, China. E-mail address: penghanwei@126.com

aMuyuan Liu, Litian Tong and Manbin Xu contributed equally to this work. 


\section{ABSTRACT}

Background: Due to the low incidence of mucoepidermoid carcinoma, there lacks sufficient studies for determining optimal treatment and predicting prognosis. The purpose of this study was to develop prognostic nomograms, to predict overall survival and disease-specific survival (DSS) of oral and oropharyngeal mucoepidermoid carcinoma patients, using the National Cancer Institute's Surveillance, Epidemiology, and End Results (SEER) database.

Methods: Clinicopathological and follow-up data of patients diagnosed with oral and oropharyngeal mucoepidermoid carcinoma between 2004 and 2017 were collected from the SEER database. The Kaplan-Meier method with the log-rank test was employed to identify single prognostic factors. Multivariate Cox regression was utilized to identify independent prognostic factors. C-index, area under the ROC curve (AUC) and calibration curves were used to assess performance of the prognostic nomograms.

Results: A total of 1230 patients with oral and oropharyngeal mucoepidermoid carcinoma were enrolled in the present study. After multivariate Cox regression analysis, age, sex, tumor subsite, $\mathrm{T}$ stage, $\mathrm{N}$ stage, $\mathrm{M}$ stage, grade and surgery were identified as independent prognostic factors for overall survival. T stage, $\mathrm{N}$ stage, $\mathrm{M}$ stage, grade and surgery were identified as independent prognostic factors for disease-specific survival. Nomograms were constructed to predict the overall survival and disease-specific survival based on the independent prognostic factors. The fitted nomograms possessed excellent prediction accuracy, with a C-index of 0.899 for OS prediction and 0.893 for DSS prediction. Internal validation by computing the bootstrap calibration plots, using the validation set, indicated excellent performance by the nomograms.

Conclusion: The prognostic nomograms developed, based on individual clinicopathological characteristics, in the present study, accurately predicted the overall survival and disease-specific survival of patients with oral and oropharyngeal mucoepidermoid carcinoma. 
Keywords: Oral cavity, Oropharynx, Mucoepidermoid carcinoma, Prognosis, Overall survival, Disease-specific survival, Nomogram, SEER 


\section{INTRODUCTION}

Mucoepidermoid carcinoma (MEC) is the most common histologic subtype of the minor salivary gland malignancies, and mainly arises from the minor salivary glands of the upper aerodigestive tract, with the oral cavity and oropharynx representing the most common anatomic locations ${ }^{1-3}$. Due to the low incidence of this tumor, studies are lacking for determining the optimal treatment, as well as predict prognosis.

The Surveillance, Epidemiology and End Results (SEER) database (https://seer.cancer.gov/statfacts/) is a publicly available resource for studies of cancer-based epidemiology, TNM staging, treatment and survival. It consists of 18 cancer registries and covers about $30 \%$ of the total US population. This database is a valuable tool for the analysis of rare cancers.

A nomogram is a visualization tool that has been widely used to predict the prognosis of cancer patients ${ }^{4-7}$. It is valuable for clinical decision-making and patient counseling. However, a prognostic nomogram for MEC arising from the oral cavity and oropharynx has not been developed. In this study, we aim to establish and validate prognostic nomograms to predict overall survival and disease-specific survival of oral and oropharyngeal MEC using data extracted from the SEER database. We expect that our study will improve the understanding of oral and oropharyngeal MEC and optimize clinical decision-making, as well as patient counseling. 


\section{MATERIALS AND METHODS}

\section{Data Source and Study Population}

All relevant data in this study were obtained from the SEER database by using SEER*Stat software version 8.3.6. We selected all cases of MEC (International Classification of Disease code: 8430 ) arising from oral cavity or oropharynx in the SEER database from 2004 to 2017. Inclusion criteria were as follows: the primary location of the tumor was the oral cavity or oropharynx; the diagnosis was histologically confirmed as MEC; clinicopathological characteristics, including age, race, sex, TNM staging, tumor subsite and surgery, were known in detail; follow-up data including survival time, vital status and cause-specific death classification were reported. Patients with missing or unknown clinicopathological data, or incomplete follow-up information were excluded in the analysis.

Demographic and clinicopathological variables extracted from the SEER database included age, race, sex, pathologic TNM status (AJCC Staging Manual, 7th edition), tumor subsite, histologic grade, surgery, survival time, vital status and cause-specific death classification. The primary outcomes were overall survival (OS) and disease-specific survival (DSS). OS was defined as the interval between the date of diagnosis and the date of death from any cause or censoring. DSS was defined as the time from diagnosis to death specific to MEC-related causes.

\section{Development and Validation of Prognostic Nomograms}

The dataset extracted from the SEER database was randomly split into $70 \%$ for the training set and $30 \%$ for the validation set. The training set was used to identify independent prognostic factors and establish nomograms, and the validation set to test the performance of the nomograms. First, survival data from the training set were analyzed using a Kaplan-Meier survival plot to identify single significant factors of survival outcomes. Then, variables with $p$-values less than 0.1 by the log-rank test were included in the multivariate Cox hazard ratio regression to select the best model for predicting overall survival and disease-specific survival of MEC. Subsequently, a prognostic risk score was calculated by combining the status of prognostic factors and their regression coefficients from the multivariate Cox regression model, and patients 
were divided into high-risk and low-risk groups according to the risk score. Then, Kaplan-Meier curves were plotted to compare the OS and DSS of low-risk and high-risk groups. Finally, nomograms for predicting the OS and DSS of oral and oropharyngeal MEC were constructed using the independent prognostic factors selected by multivariate Cox regression.

The area under the ROC curve (AUC) and C-index were calculated to assess the predictive power of the prognostic models. In addition, calibration curves were drawn based on the bootstrap method to assess the performance of the prognostic nomograms.

\section{Statistical Analysis}

All statistical analyses were performed with $\mathrm{R}$ software (Version 4.0.2). The Kaplan-Meier method was used to identify single significant prognostic factors of survival outcomes. The variables found to be statistically significant by log rank test in survival analysis were included in the multivariate Cox hazard ratio regression to screen for independent prognostic factors. Then, prognostic nomograms for predicting overall survival (OS) and disease-specific survival (DSS) were developed by R software using the "rms" package. The predictive capacity of the prognostic nomograms was estimated by using a calibration curve, the concordance index (C-index) and the area under the ROC curve (AUC). A time-dependent receiver operating characteristic (ROC) curve was constructed by using the "survivalROC" package. The calibration curve was plotted using "rms", "foreign" and "survival" packages. All significance tests were two-sided, and a $P<0.05$ was considered significant. 


\section{RESULTS}

\section{Characteristics of the Study Cohort}

We identified 1230 oral and oropharyngeal MEC patients that met our inclusion criteria. The median follow-up time for the cohort was 66 months. The median age at diagnosis was 53 years. Among the 1230 patients, 524 (42.6\%) were men and 706 $(57.4 \%)$ were women. With respect to race, 935 (76.0\%) patients were white, 156 $(12.7 \%)$ were black and $139(11.3 \%)$ were of other races (Including American Indian/Alaskan Natives and Asian/Pacific Islanders). For TNM status, 828 (67.3\%) patients were AJCC stage I, 165 (13.4\%) patients were stage II, 62 (5.1\%) patients were stage III and 175 (14.2\%) patients were stage IV. For histological grade, 333 (27.1\%) patients had well differentiated, 658 (53.5\%) patients had moderately differentiated, 149 (12.1\%) patients had poorly differentiated or undifferentiated tumors, and 90 (7.3\%) patients had tumors of unknown grade. The 1230 patients were randomly divided into a training set $(n=862)$ and validation set $(n=368)$. The clinicopathological characteristics and comparison of the 2 cohorts are summarized in Table 1. In addition, the chi-square test showed the absence of significant deviation in distribution between the training and validation sets.

\section{Survival Analysis and Prognostic Factor Identification}

We employed Kaplan-Meier analysis to identify single significant prognostic factors in the training set. Our results showed that the 3-year, 5-year and 10-year OSs were $90.8 \%, 87.2 \%$ and $80.8 \%$, respectively. The 3-year, 5-year, and 10-year DSSs were $93.8 \%, 92.4 \%$ and $90.2 \%$, respectively. The influence of the following factors on OS and DSS of oral and oropharyngeal MEC was investigated: age, sex, race, AJCC TNM status, tumor subsite, histologic grade and treatment. With respect to overall survival analysis, all the factors mentioned above yielded a $p$-value less than 0.1 by the log-rank test (Fig. 1). For disease-specific survival analysis, all the factors described above, except for race, yielded a $p$-value less than 0.1 by log-rank test (Fig. 2). Then, related prognostic factors with a $p$-value less than 0.1 by the log-rank test were adjusted for multivariate analysis. The forest plots of the multivariate Cox regression for OS and DSS are shown in Fig. 3A and Fig. 4A, respectively. After 
multivariate analysis, age, sex, race, TNM status, tumor subsite, grade and surgery were identified as independent prognostic factors for OS $(p<0.05)$. For DSS, only T stage, $\mathrm{N}$ stage, M stage, grade and surgery remained independent factors. Then, based on the prognostic model score, MEC patients in the training cohort were stratified into a high-risk group and low-risk group, and Kaplan-Meier survival curves were plotted to compare the overall survival and disease-specific survival of the model-predicted risk groups. Results strongly demonstrated that patients in the high-risk group had significantly poorer overall or disease-specific survival than patients in the low-risk group ( $p<0.0001)$ (Fig.5).

\section{Prognostic Nomogram Development and Validation}

In the current study, two prognostic nomograms were developed by using the independent prognostic factors identified by multivariate Cox regression (OS nomogram is shown in Fig. 3B and DSS nomogram is shown in Fig. 4B). Based on the total score generated by adding the score obtained from individual characteristics, clinicians can conveniently predict the 3-year, 5-year and 10-year OS and DSS. The C-index was 0.89 for OS prediction and 0.95 for DSS prediction, implying near perfect prediction accuracy of the fitted models. In addition, the area under receiver operating characteristic curve (ROC AUC) showed that our OS and DSS nomograms provided excellent prediction performance (3-year OS AUC =0.905, 5-year OS AUC=0.894, 10-year OS AUC=0.883, 3-year DSS AUC=0.981, 5-year DSS AUC=0.964 and 10-year DSS AUC=0.943) (Fig. 6). Finally, the calibration plot-predicted 3-year, 5-year and 10-year OS and DSS of MEC, built to internally validate the OS prediction model and DSS prediction model, performed very well compared with the ideal curve (Fig.7).

The validation set was used to internally validate the accuracy and reliability of the established nomograms. First, the discrimination ability of the nomograms was assessed using a C-index. In the current study, the C-index was 0.899 for the OS prediction nomogram and 0.893 for the DSS prediction nomogram, indicating excellent prediction ability of the fitted nomograms. Second, the area under receiver operating characteristic curve (ROC AUC) demonstrated that the fitted OS and DSS 
nomograms provided near perfect prediction performance (3-year OS AUC $=0.906$, 5-year OS AUC=0.918, 10-year OS AUC=0.901, 3-year DSS AUC=0.933, 5-year DSS AUC=0.925 and 10-year DSS AUC=0.882) (Fig. 8). Third, calibration curves based on the bootstrap resampling method were plotted. In comparison to the ideal model, the calibration curves for 3-year, 5-year, 10-year OS and DSS were excellent predictors (Fig. 9). Finally, all patients in the validation set were classified into high-risk and low-risk groups by the risk score calculated using the OS and DSS prediction models. Similar to the training set, survival analysis showed that patients in the high-risk group had worse OS and DSS than those in the low-risk group in the validation set (Fig. 10, $p<0.0001)$. 


\section{DISCUSSION}

In the current study, we developed and validated two prognostic nomograms, one for predicting overall survival and the other for predicting disease-specific survival of oral and oropharyngeal MEC. Clinicians can conveniently predict the 3-year, 5-year and 10-year OS or DSS of MEC patients based on the sum of all scores obtained from individual prognostic factors in the nomograms. To the best of our knowledge, this is the first study to establish prognostic nomograms for MEC of the oral cavity and oropharynx. The fitted nomograms possess excellent prediction accuracy, with a C-index of 0.899 for OS prediction and 0.893 for DSS prediction, a 3-year OS AUC of 0.906, 5-year OS AUC of 0.918, 10-year OS AUC of 0.901, 3-year DSS AUC of 0.933, 5-year DSS AUC of 0.925 and 10-year DSS AUC of 0.882. Furthermore, internal validation by computing the bootstrap calibration plots using the validation set indicated excellent performance by the nomograms.

In recent years, more and more prognostic nomograms have been constructed and utilized for prediction of prognosis and clinical decision-making for different types of carcinomas 5,8-12. However, because of the low incidence of mucoepidermoid carcinoma, research focusing on this tumor is very rare. In 2014, after conducting a survival analysis of 45 patients with salivary MEC, Yamazaki et al. ${ }^{3}$ reported a 5-year disease-specific survival rate of $81.8 \%$ and tried to identify clinicopathological factors strongly associated with prognosis and outcome. However, the number of cases in their study was too small to draw any conclusions. In 2017, Goel et al. ${ }^{13}$ conducted a population-based analysis of 1426 patients with minor salivary gland carcinoma of the oropharynx and demonstrated that tumor grade, $\mathrm{T}$ stage, $\mathrm{N}$ stage and age were independent prognosticators. They reported 5-year and 10-year disease-specific survival rates of $75.1 \%$ and $61.6 \%$, respectively. However, minor salivary gland carcinomas include a multitude of histologic subtypes, such as mucoepidermoid carcinoma, adenoid cystic carcinoma and adenocarcinoma, and each histologic subtype has its own biological behavior $2,14,15$. Hence, it is not suitable to analyze minor salivary gland carcinoma as a single tumor type. In addition, no prognostic nomogram has been established in prior studies. In 2019, Jian et al. ${ }^{16}$ established a 
prognostic nomogram with a $\mathrm{C}$-index of 0.877 to predict the prognosis of patients with parotid gland mucoepidermoid carcinoma, based on a SEER database study of 1306 cases. In their study, the 5-year DSS and 10-year DSS were $88.7 \%$ and $86.4 \%$, respectively, and factors including age, grade, $\mathrm{T}$ stage, $\mathrm{N}$ stage, $\mathrm{M}$ stage, chemotherapy and surgery were regarded as independent prognostic factors for DSS. However, it is well known that tumor behavior always varies by anatomical site. Oral cavity and oropharynx represent the most common anatomic locations of MEC, but unfortunately, no relevant prognostic nomogram has been developed specifically for oral and oropharyngeal MEC.

There are several advantages in the present study. First, our nomograms focus only on one histological subtype of minor salivary gland carcinoma arising from the oral cavity or oropharynx, which enables greater accuracy and reliability of our nomograms. Specifically, our prognostic nomograms yielded a very high C-index ( 0.899 for OS prediction and 0.893 for DSS prediction) and AUC, indicating excellent prognostic prediction ability compared to previous models ${ }^{3,13,16}$. Last but not least, one independent validation set and three validation methods were utilized in the current study to guarantee stability and reliability of our prognostic nomograms.

Several limitations in this study should be considered. First, the SEER database does not contain certain important clinicopathological information, such as depth of invasion, surgical margins, perineural invasion, and lymphovascular invasion. Second, although three different methods had been utilized for internal validation of our prognostic nomograms, an appropriate cohort for external validation unfortunately was not available. An external validating set will be needed to confirm our findings in the future. Third, the current study is a retrospective analysis. Further prospective research in larger cohorts will be necessary to get a more precise and reliable prediction model. 


\section{CONCLUSIONS}

In conclusion, we developed and validated two prognostic nomograms to predict OS and DSS for patients with oral and oropharyngeal mucoepidermoid carcinoma. These prognostic nomograms ought to be valuable in clinical decision-making and patient counseling. 


\section{Conflict of interest statement}

The authors declare that they have no conflicts of interest to this work. 


\section{Funding}

This project was supported by the National Natural Science Foundation of China (NSFC) (Grant No. 31770876). the Guangdong Medical Research Foundation, China (Grant No. A2019424) and the Shantou Science and Technology Project, Guangdong Province, China (Grant No. 180723154011142). 


\section{Author contribution statement}

All authors contributed to the design of the study. MYL, LTT, MBX, HWP and DYH contributed in acquisition of data, manuscript preparation and revision. MYL, LTT, MBX, HWP and DYH participated in study planning, data interpretation, and writing the manuscript. All authors reviewed and approved the final version of the manuscript. 


\section{Tables}

Table 1. Clinicopathological Parameters of the Training Set and Validation Set in Oral and Oropharyngeal Mucoepidermoid Carcinoma. 


\section{Figures}

Figure 1. Kaplan-Meier curves for overall survival by different clinicopathological factors. (A) Age; (B) race; (C) sex; (D) T stage; (E) N stage; (F) M stage; (G) tumor subsite; $(\mathrm{H})$ grade; (I) surgery.

Abbreviation: $\mathbf{A T}=$ anterior of tongue; $\mathbf{B M}=$ buccal mucuso; $\mathbf{F M}=$ floor of mouth; $\mathbf{H P}=$ hard plate; $\mathbf{G}=$ gum; $\mathbf{L}=$ lip; $\mathbf{O L}=$ Overlapping Lesion of oral cavity and oropharynx; $\mathbf{S P}=$ Soft plate; $\mathbf{T O}$ = tonsil; $\mathbf{B T}$ = Base of tongue; $\mathbf{O O}$ = Overlapping lesion of oropharynx.

Figure 2. Kaplan-Meier curves for disease-specific survival by different clinicopathological factors. (A) Age; (B) race; (C) sex; (D) T stage; (E) N stage; (F) M stage; (G) tumor subsite; $(\mathrm{H})$ grade; (I) surgery.

Abbreviation: $\mathbf{A T}=$ anterior of tongue; $\mathbf{B M}=$ buccal mucuso; $\mathbf{F M}=$ floor of mouth; $\mathbf{H P}=$ hard plate; $\mathbf{G}=$ gum; $\mathbf{L}=$ lip; $\mathbf{O L}=$ Overlapping Lesion of oral cavity and oropharynx; $\mathbf{S P}=$ Soft plate; $\mathbf{T O}=$ tonsil; $\mathbf{B T}=$ Base of tongue; $\mathbf{O O}=$ Overlapping lesion of oropharynx .

Figure 3. (A) Multivariate Cox proportional hazard regression model and (B) nomogram for predicting overall survival.

Figure 4. (A) Multivariate Cox proportional hazard regression model and (B) nomogram for predicting disease-specific survival.

Figure 5. Kaplan-Meier plots by risk group for the training set. (A) Kaplan-Meier plot for overall survival and (B) Kaplan-Meier plot for disease-specific survival.

Figure 6. Time-dependent ROC curves for predicting the (A) 3-year, (B) 5-year, (C) 10-year overall survival, and (D) 3-year, (E) 5-year and (F) 10-year disease-specific survival.

Figure 7. Calibration curves of the prognostic nomograms for the training set. $(A)$ 3-year, (B) 5-year and (C) 10-year overall survival nomogram calibration curves; (D) 3-year, (E) 5-year and (F) 10-year disease-specific survival nomogram calibration curves. The $\mathrm{X}$-axis represents the model-predicted survival, and the $\mathrm{Y}$-axis represents the actual survival. The dotted line represents the ideal model.

Figure 8. Time-dependent ROC curves for the validation set. Prediction for (A) 3-year 
overall survival, (B) 5-year overall survival, (C) 10-year overall survival and (D) 3-year disease-specific survival, (E) 5-year disease-specific survival and (F) 10-year disease-specific survival.

Figure 9. Calibration curves for the validation set. (A) 3-year, (B) 5-year and (C) 10-year overall survival validation calibration curves; (D) 3-year, (B) 5-year and (C) 10-year disease-specific survival validation calibration curves. The X-axis represents the model-predicted survival, and the $\mathrm{Y}$-axis represents the actual survival. The dotted line represents the ideal model.

Figure 10. Kaplan-Meier plots by risk group for the validation set. (A) Kaplan-Meier curves for overall survival and (B) Kaplan-Meier curves for disease-specific survival. 


\section{References}

1 Strick, M. J., Kelly, C., Soames, J. V. \& McLean, N. R. Malignant tumours of the minor salivary glands--a 20 year review. Br J Plast Surg 57, 624-631, doi:10.1016/j.bjps.2004.04.017 (2004).

2 Baddour, H. M., Jr., Fedewa, S. A. \& Chen, A. Y. Five- and 10-Year Cause-Specific Survival Rates in Carcinoma of the Minor Salivary Gland. JAMA Otolaryngol Head Neck Surg 142, 67-73, doi:10.1001/jamaoto.2015.2805 (2016).

3 Yamazaki, K., Ohta, H., Shodo, R., Matsuyama, H. \& Takahashi, S. Clinicopathological features of mucoepidermoid carcinoma. J Laryngol Otol 128, 91-95, doi:10.1017/S0022215113003459 (2014).

$4 \quad \mathrm{Li}, \mathrm{Y}$. et al. Nomograms to estimate long-term overall survival and tongue cancer-specific survival of patients with tongue squamous cell carcinoma. Cancer Med 6, 1002-1013, doi:10.1002/cam4.1021 (2017).

5 Low, S. K. et al. Prognostic factors and nomogram for survival prediction in patients with primary pulmonary lymphoma: a SEER population-based study. Leuk Lymphoma 60, 3406-3416, doi:10.1080/10428194.2019.1633636 (2019).

6 Montero, P. H. et al. Nomograms for preoperative prediction of prognosis in patients with oral cavity squamous cell carcinoma. Cancer 120, 214-221, doi:10.1002/cncr.28407 (2014).

7 Sun, J. et al. Nomogram to predict the prognosis of parotid gland mucoepidermoid carcinoma: a population-based study of 1306 cases. PeerJ 7, e7237, doi:10.7717/peerj.7237 (2019).

8 Lin, S. et al. Development and validation of a nomogram for predicting survival of advanced breast cancer patients in China. Breast 53, 172-180, doi:10.1016/j.breast.2020.08.004 (2020).

Qiu, Q. et al. Development and Validation of a Radiomics Nomogram Model for Predicting Postoperative Recurrence in Patients With Esophageal Squamous Cell Cancer Who Achieved pCR After Neoadjuvant Chemoradiotherapy Followed by Surgery. Front Oncol 10, 1398, doi:10.3389/fonc.2020.01398 (2020).

Shi, X., Xu, L., Ma, B. \& Wang, S. Development and validation of a nomogram to predict the prognosis of patients with gastric cardia cancer. Sci Rep 10, 14143, doi:10.1038/s41598-020-71146-z (2020).

11 Wu, J. et al. Prognostic nomogram for predicting survival in patients with high grade endometrial stromal sarcoma: a Surveillance Epidemiology, and End Results database analysis. Int J Gynecol Cancer, doi:10.1136/ijgc-2020-001409 (2020).

Wu, Y. et al. An Externally-Validated Dynamic Nomogram Based on Clinicopathological Characteristics for Evaluating the Risk of Lymph Node Metastasis in Small-Size Non-small Cell Lung Cancer. Front Oncol 10, 1322, doi:10.3389/fonc.2020.01322 (2020).

13 Goel, A. N., Badran, K. W., Braun, A. P. G., Garrett, A. M. \& Long, J. L. Minor Salivary Gland Carcinoma of the Oropharynx: A Population-Based Analysis of 1426 Patients. Otolaryngol Head Neck Surg 158, 287-294, doi:10.1177/0194599817735309 (2018).

Kakarala, K. \& Bhattacharyya, N. Survival in oral cavity minor salivary gland carcinoma. Otolaryngol Head Neck Surg 143, 122-126, doi:10.1016/j.otohns.2010.02.033 (2010).

$\mathrm{Li}, \mathrm{Q}$. et al. Long-term treatment outcome of minor salivary gland carcinoma of the hard palate. Oral Oncol 48, 456-462, doi:10.1016/j.oraloncology.2011.12.005 (2012). Case Rep 2015, doi:10.1136/bcr-2014-208339 (2015). 
Figures
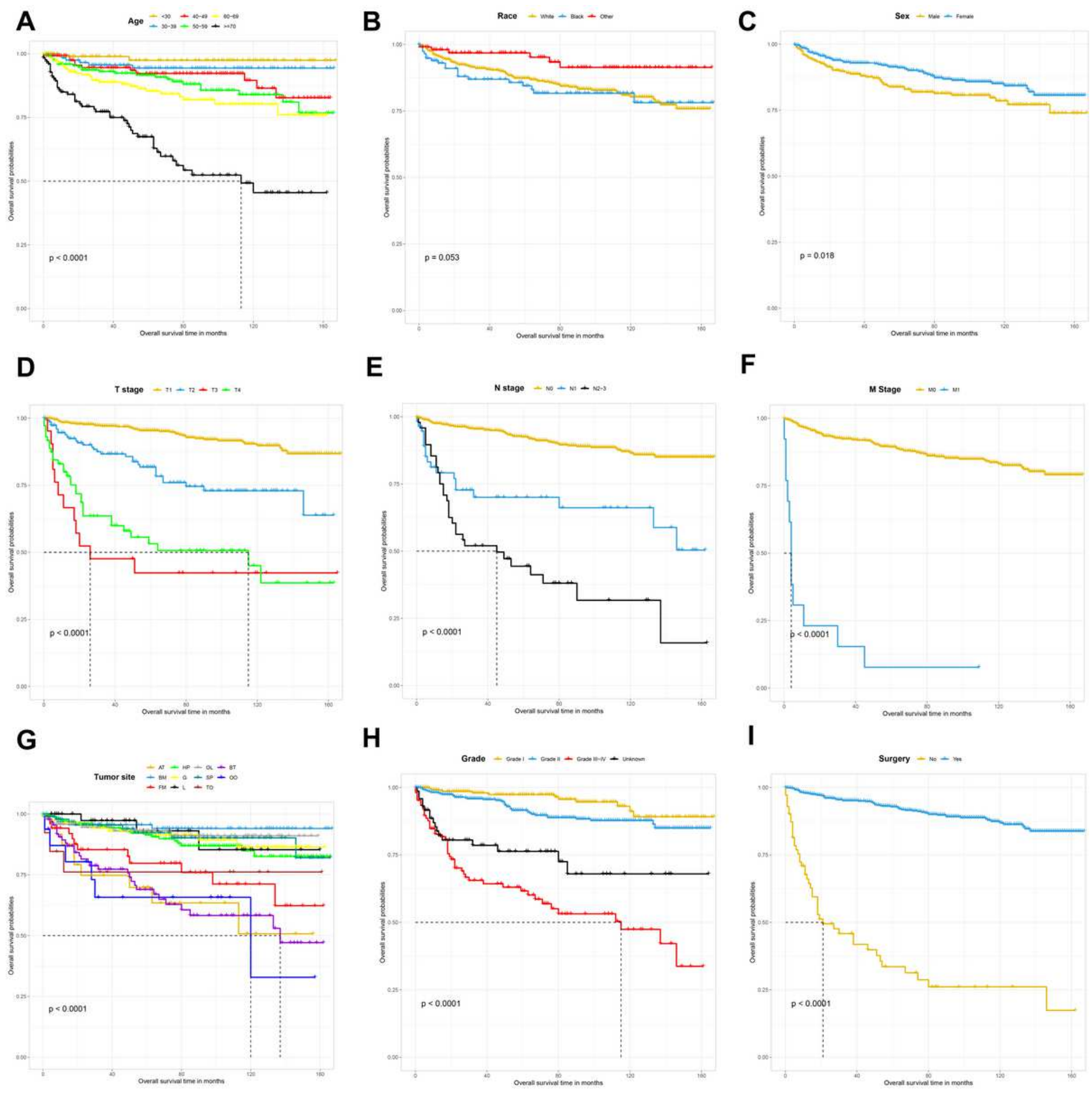

Figure 1

Kaplan-Meier curves for overall survival by different clinicopathological factors. (A) Age; (B) race; (C) sex; (D) T stage; (E) N stage; (F) M stage; (G) tumor subsite; (H) grade; (I) surgery. Abbreviation $\mathbb{A} A T$ = anterior of tongue; $\mathrm{BM}$ = buccal mucuso; $\mathrm{FM}=$ floor of mouth; $\mathrm{HP}=$ hard plate; $\mathrm{G}=$ gum; $\mathrm{L}=$ lip; $\mathrm{OL}=$ Overlapping 
Lesion of oral cavity and oropharynx; $\mathrm{SP}=$ Soft plate; $\mathrm{TO}=$ tonsil; $\mathrm{BT}=$ Base of tongue; $\mathrm{OO}=$ Overlapping lesion of oropharynx.

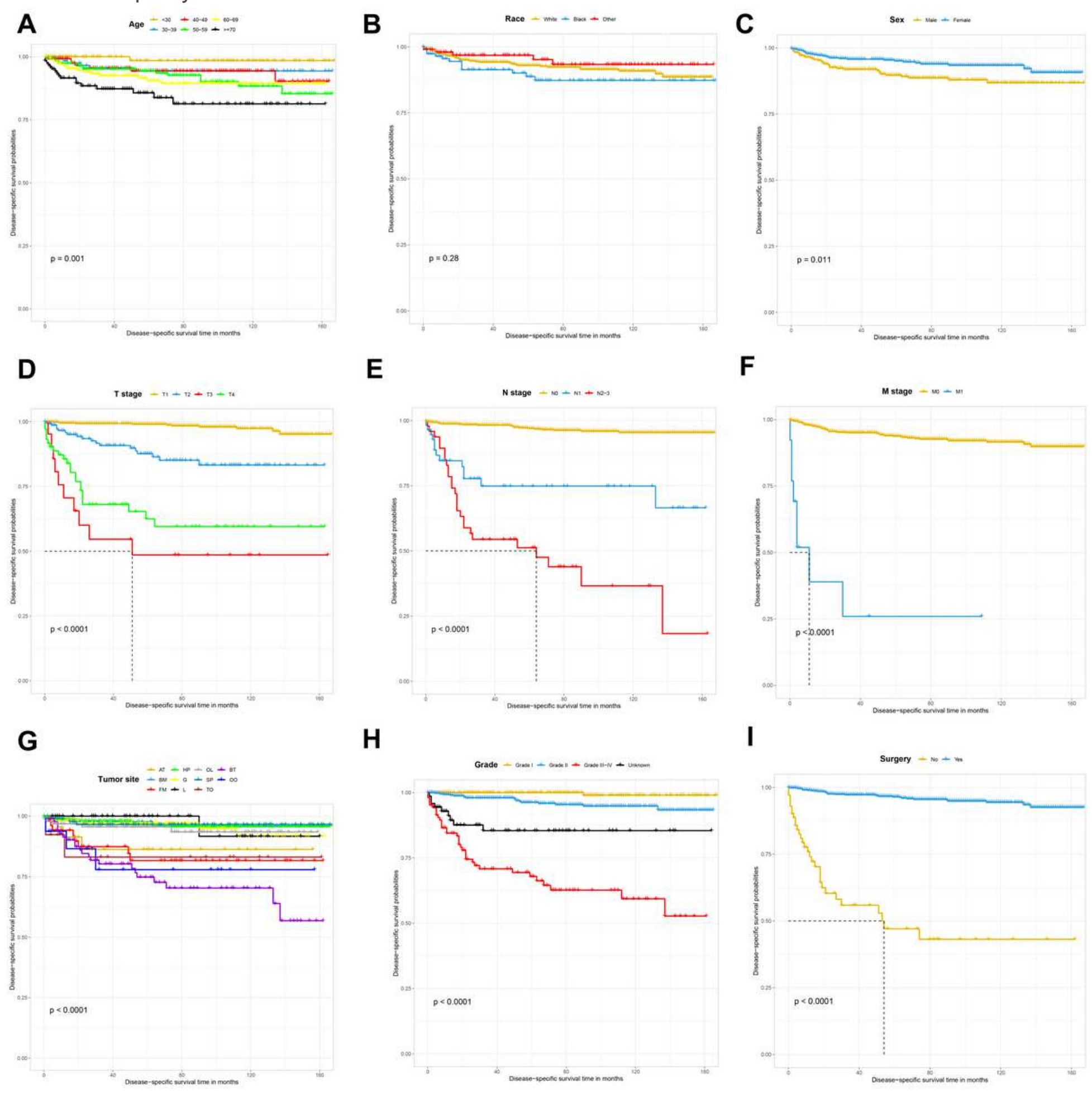

\section{Figure 2}

Kaplan-Meier curves for disease-specific survival by different clinicopathological factors. (A) Age; (B)

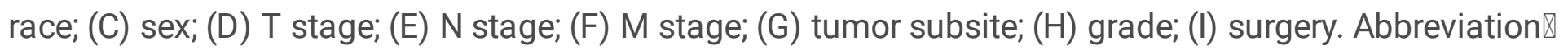
$A T=$ anterior of tongue; $B M=$ buccal mucuso; $F M=$ floor of mouth; $H P=$ hard plate; $G$ = gum; $L=$ lip; $O L=$ 
Overlapping Lesion of oral cavity and oropharynx; SP = Soft plate; TO = tonsil; BT = Base of tongue; OO = Overlapping lesion of oropharynx.
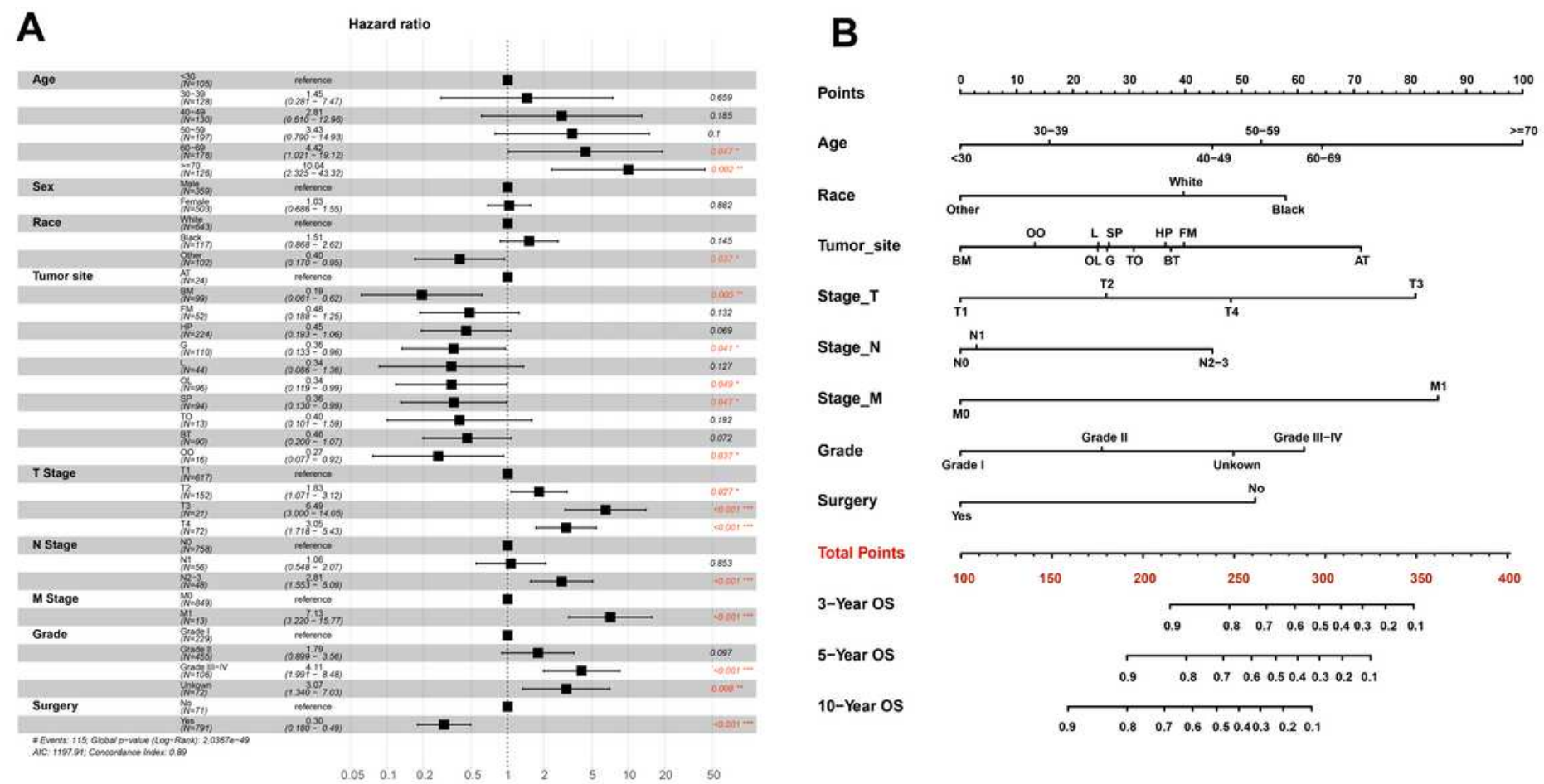

Figure 3

(A) Multivariate Cox proportional hazard regression model and (B) nomogram for predicting overall survival.

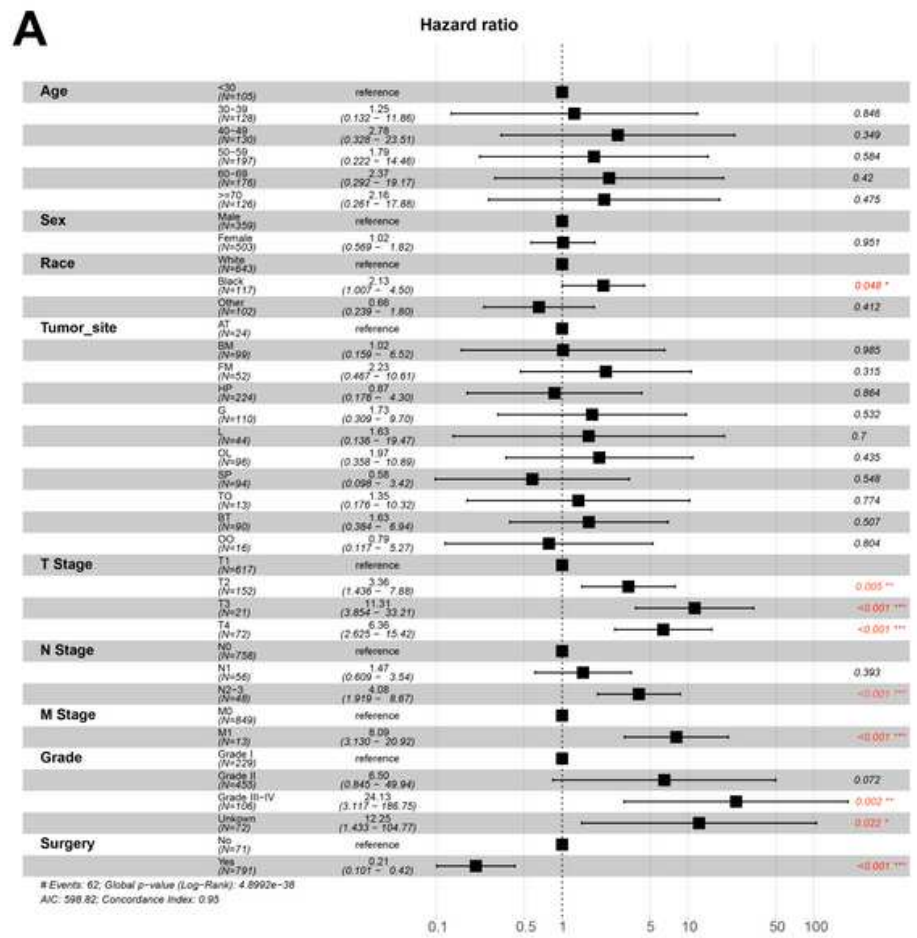

\section{B}

Points

Race

T Stage

N Stage

M Stage

Grade

Surgery

Total Points

3-Year DS

5-Year DS

10-Year DS
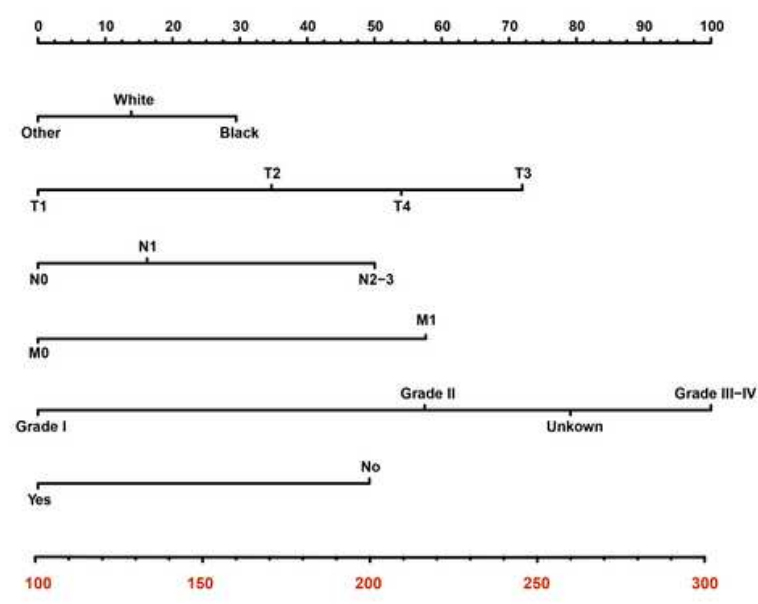

\begin{tabular}{lllllllllll}
\hline 0.9 & 0.8 & 0.7 & 0.6 & 0.5 & 0.4 & 0.3 & 0.2 & 0.1
\end{tabular}

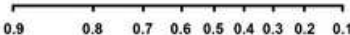

\begin{tabular}{llllllllll}
\hline 0.9 & 0.8 & 0.7 & 0.6 & 0.5 & 0.4 & 0.3 & 0.2 & 0.1
\end{tabular} 
Figure 4

(A) Multivariate Cox proportional hazard regression model and (B) nomogram for predicting diseasespecific survival.
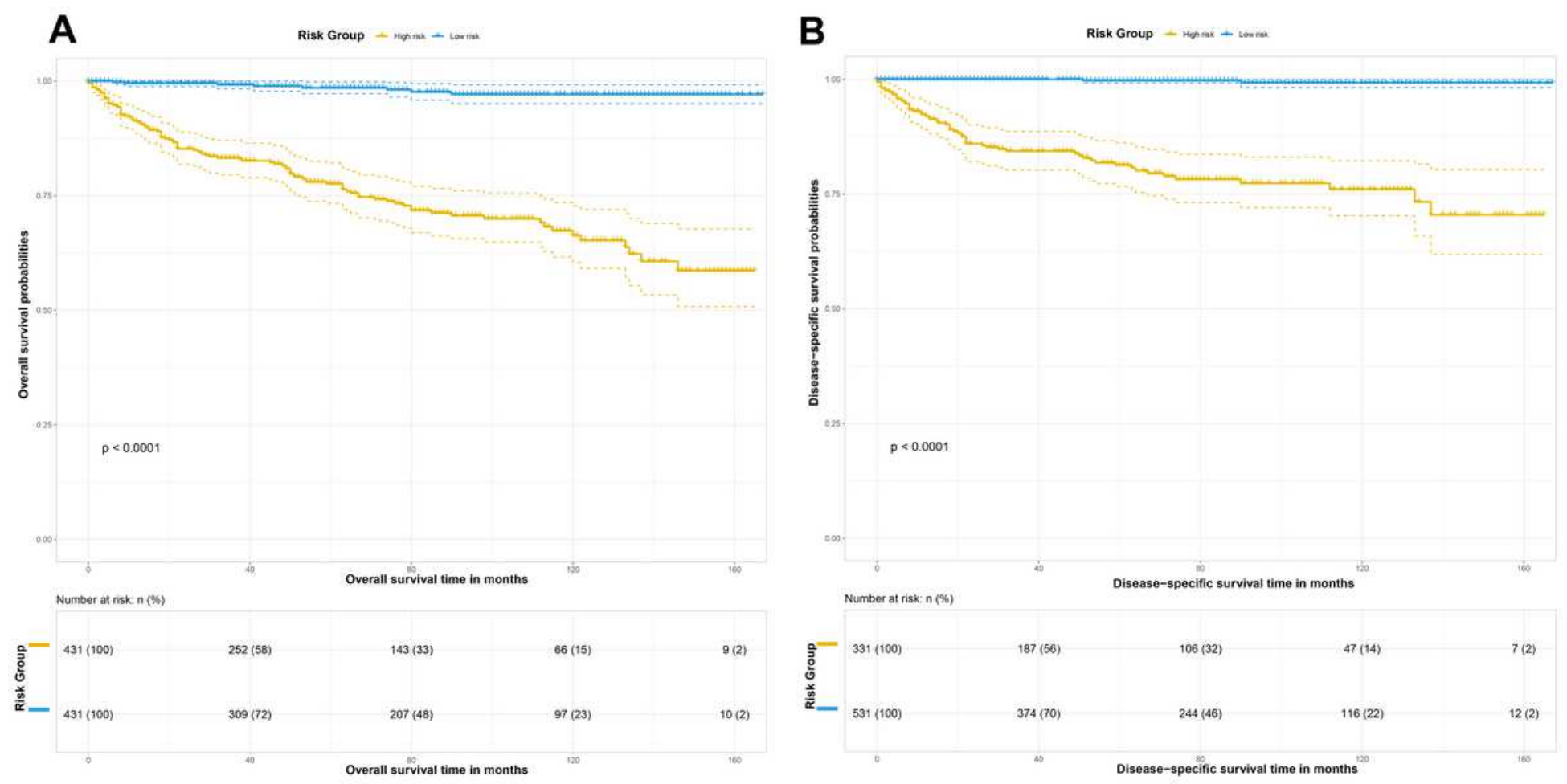

Figure 5

Kaplan-Meier plots by risk group for the training set. (A) Kaplan-Meier plot for overall survival and (B) Kaplan-Meier plot for disease-specific survival. 

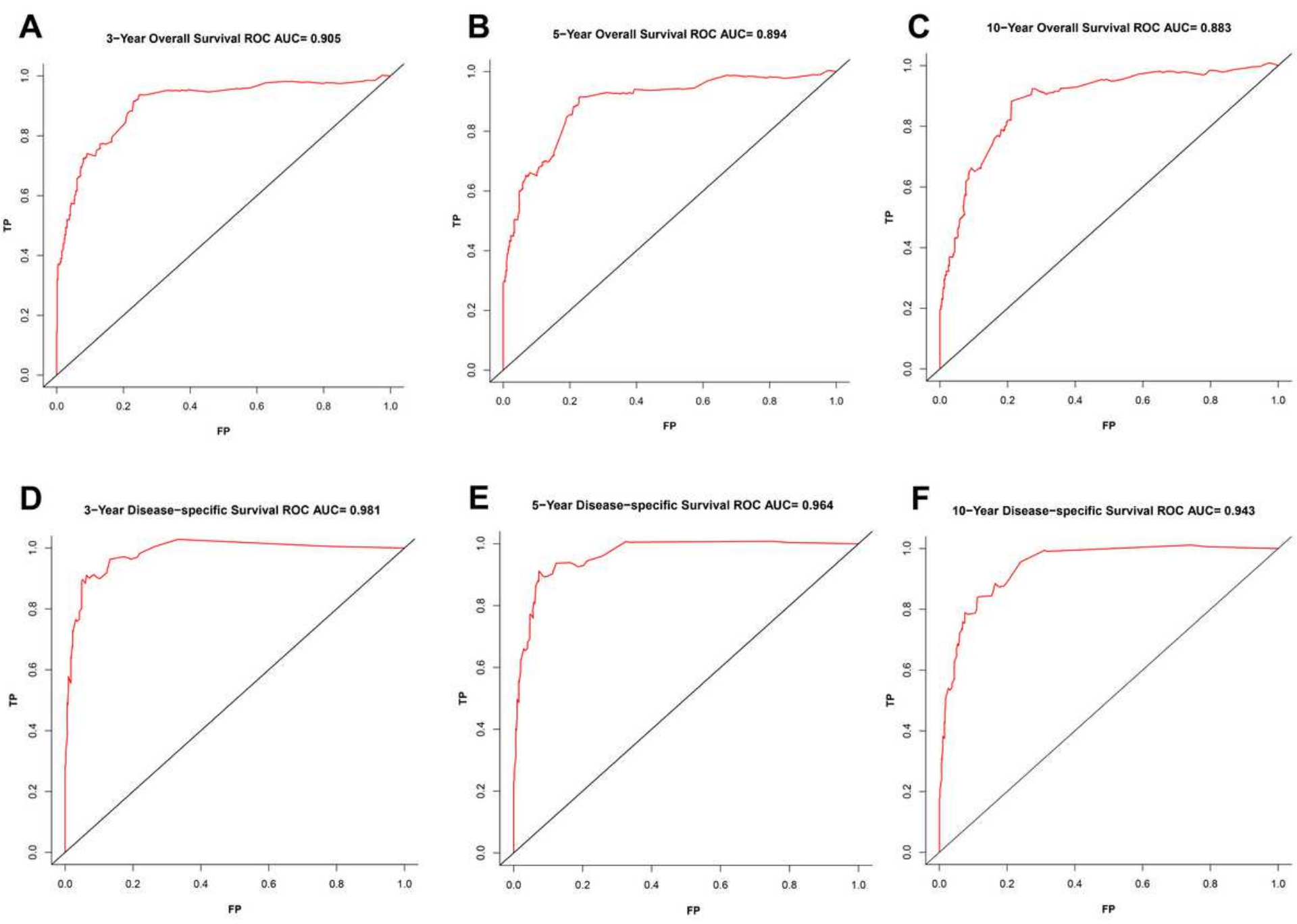

\section{Figure 6}

Time-dependent ROC curves for predicting the (A) 3-year, (B) 5-year, (C) 10-year overall survival, and (D) 3year, (E) 5-year and (F) 10-year disease-specific survival. 
A
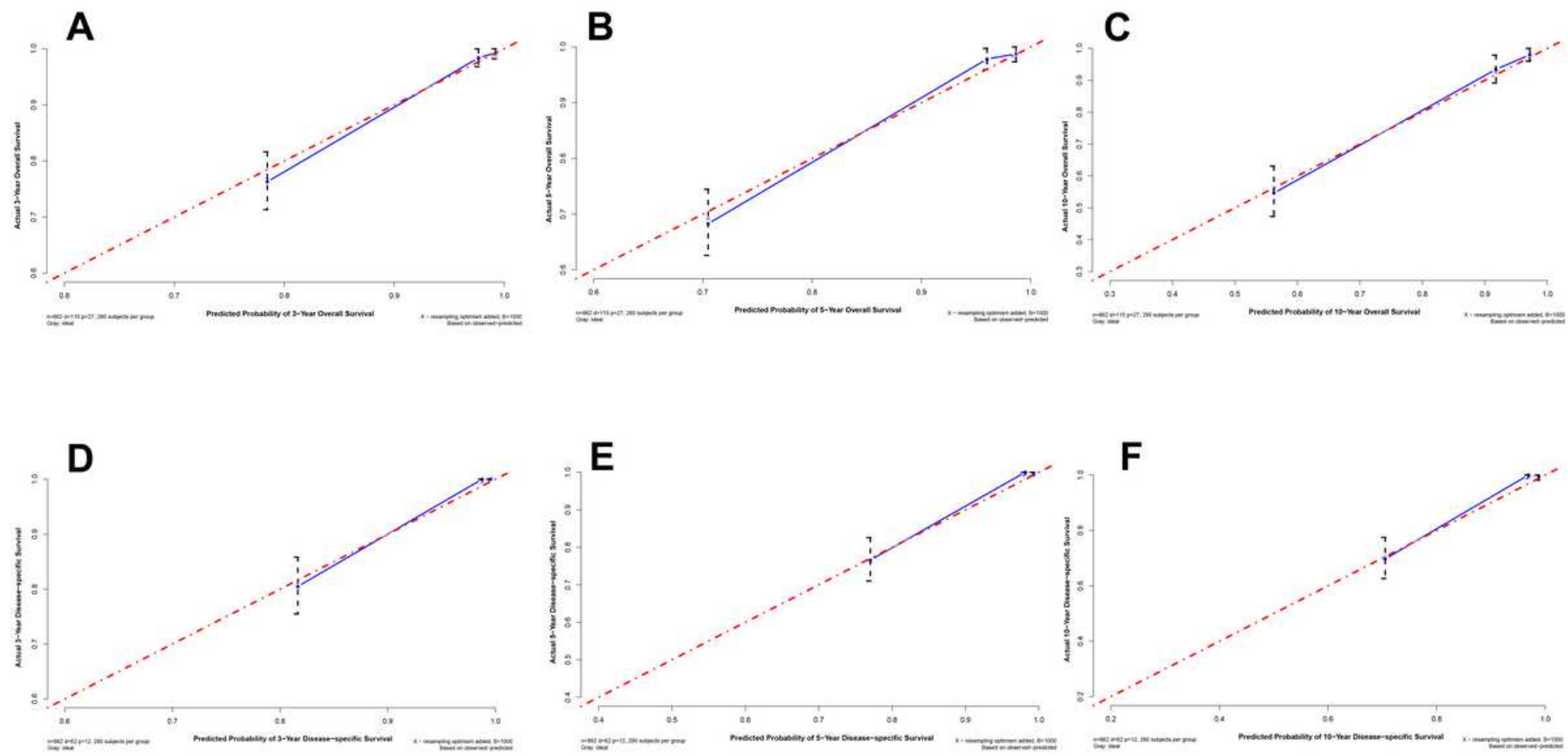

\section{Figure 7}

Calibration curves of the prognostic nomograms for the training set. (A) 3-year, (B) 5-year and (C) 10-year overall survival nomogram calibration curves; (D) 3-year, (E) 5-year and (F) 10-year disease-specific survival nomogram calibration curves. The $\mathrm{X}$-axis represents the model-predicted survival, and the $\mathrm{Y}$-axis represents the actual survival. The dotted line represents the ideal model. 

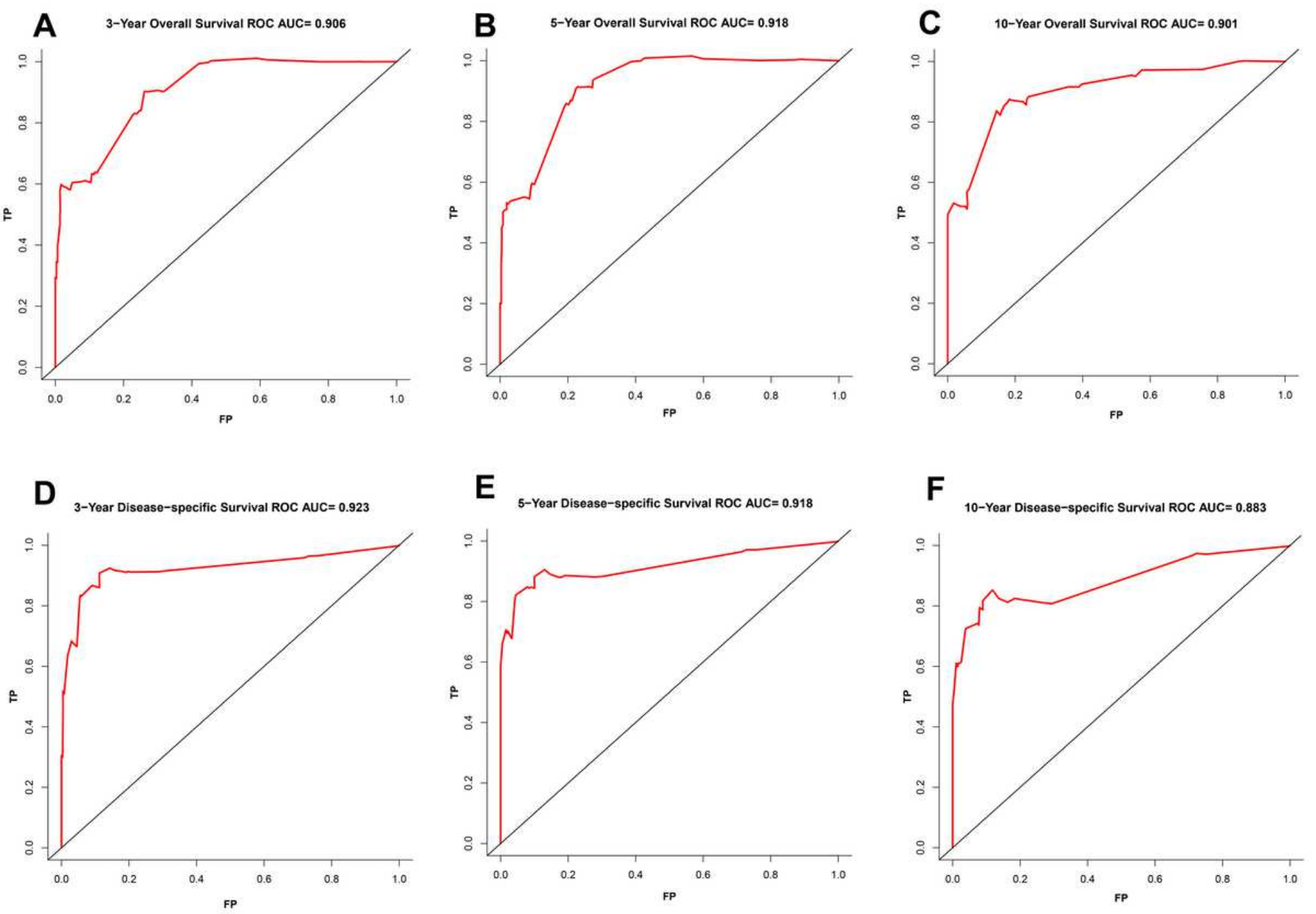

Figure 8

Time-dependent ROC curves for the validation set. Prediction for (A) 3-year overall survival, (B) 5-year overall survival, (C) 10-year overall survival and (D) 3-year disease-specific survival, (E) 5-year diseasespecific survival and (F) 10-year disease-specific survival. 
A

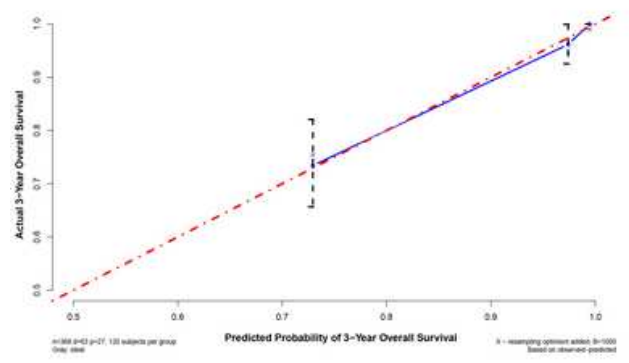

D

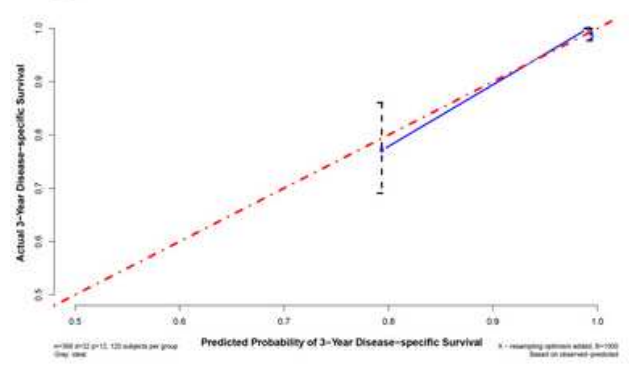

B

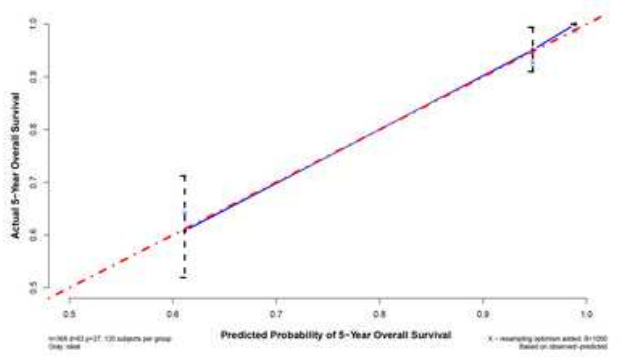

E

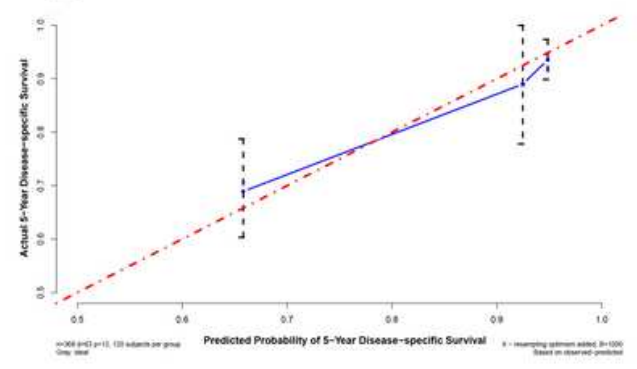

C

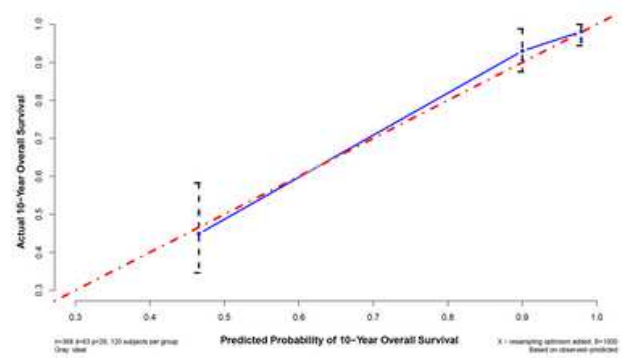

$\mathbf{F}$

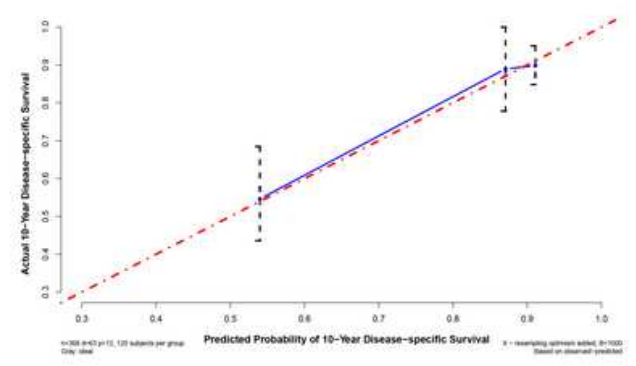

Figure 9

Calibration curves for the validation set. (A) 3-year, (B) 5-year and (C) 10-year overall survival validation calibration curves; (D) 3-year, (B) 5-year and (C) 10-year disease-specific survival validation calibration curves. The $\mathrm{X}$-axis represents the model-predicted survival, and the $\mathrm{Y}$-axis represents the actual survival. The dotted line represents the ideal model.

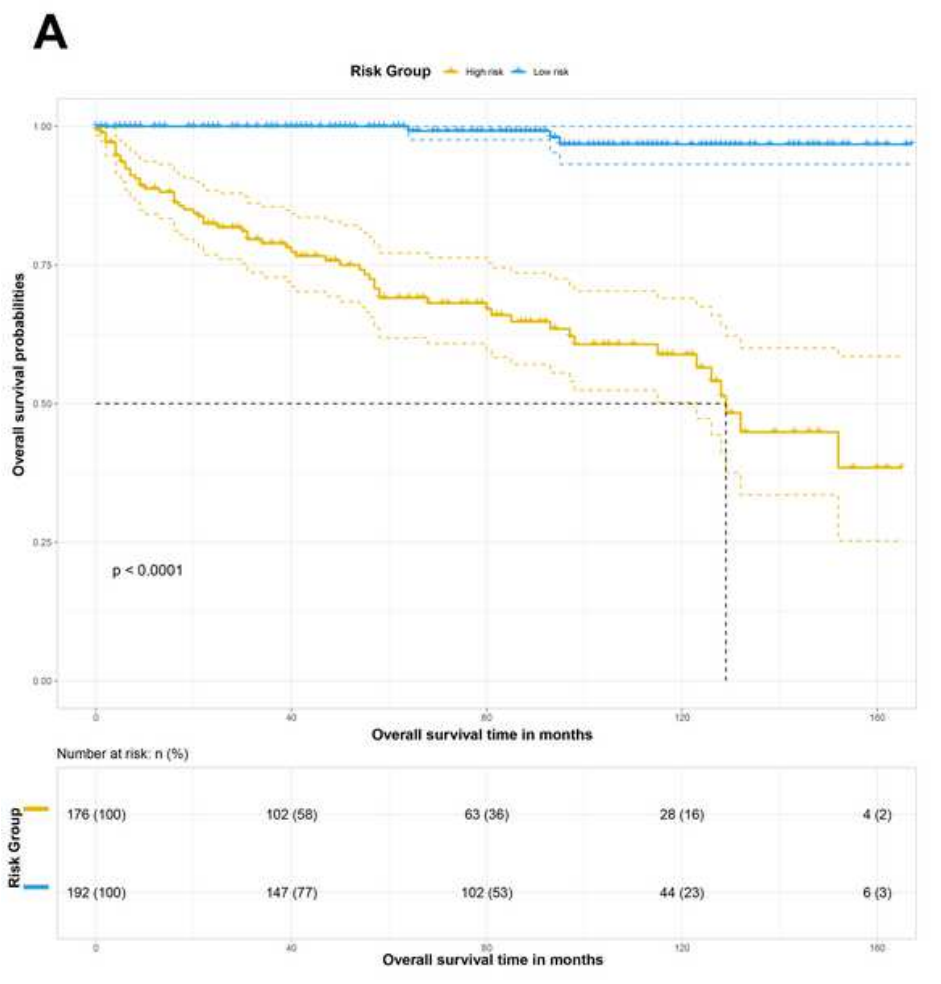

B

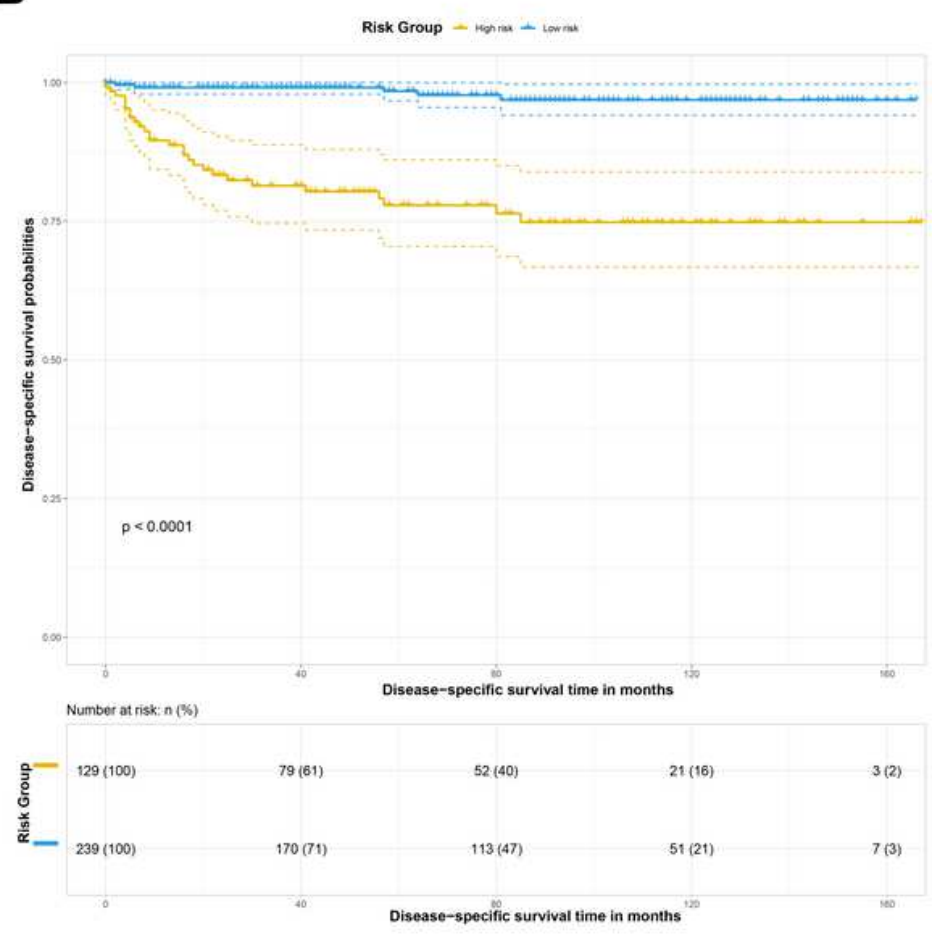


Figure 10

Kaplan-Meier plots by risk group for the validation set. (A) Kaplan-Meier curves for overall survival and (B) Kaplan-Meier curves for disease-specific survival.

\section{Supplementary Files}

This is a list of supplementary files associated with this preprint. Click to download.

- Table1.docx 